\title{
Detection of buried objects using reflected GNSS signals
}

\author{
Riccardo Notarpietro ${ }^{*}$, Salvatore De Mattia, Maurizio Campanella, Yuekun Pei and Patrizia Savi
}

\begin{abstract}
The use of reflected Global Navigation Satellite System (GNSS) signals for sensing the Earth has been growing rapidly in recent years. This technique is founded on the basic principle of detecting GNSS signals after they have been reflected off the Earth's surface and using them to determine the properties of the reflecting surface remotely. This is the so-called GNSS reflectometry (GNSS-R) technique. In this paper, a new application regarding the detection of metallic buried objects is analyzed and it is validated through several experimental campaigns. Although the penetration depth of GNSS signals into the ground is not optimal and depends on the soil moisture, GNSS signals can likely interact approximately with the first $10 \mathrm{~cm}$ of the ground and therefore can be reflected back by any metallic object buried on the first terrain layer. A very light and low-cost GNSS receiver prototype based on a software-defined radio approach was developed. This receiver can be used as a payload on board small drones or unmanned aerial systems to detect metallic objects (mines or other explosive devices). A signal processing tool based on an open-loop GNSS signal acquisition strategy was developed. The results of two experiments which show the possibility of using GNSS-R signals to detect buried metallic objects and to provide an estimate of their dimensions are discussed.
\end{abstract}

Keywords: GNSS reflectometry; Software-defined radio; Signal to noise ratio

\section{Introduction}

Remote sensing using Global Navigation Satellite System (GNSS) signals (which include, for example, the US GPS and its updates, the Russian GLONASS, the future European Galileo, the Chinese COMPASS) has become more and more popular in the last few decades to analyze the characteristics of the electromagnetic waves in the media in which they propagate in. Applications for water vapor monitoring and atmospheric/ionospheric profiling are nowadays operatively adopted (a review is provided in [1] for ground-based applications and in [2] for space-based applications). Another application has recently emerged: the use of reflected GNSS signals to extract information about the Earth's surface, named GNSS reflectometry (GNSS-R) [3,4].

The concept was first put forward as an alternative technique for ocean altimetry [5]. Later, the same principle was demonstrated as a useful tool to sense ocean roughness [6]. Exploiting a bistatic geometry approach, the GNSS satellites act as transmitters while an aircraft or a

\footnotetext{
* Correspondence: riccardo.notarpietro@polito.it

Electronic and Telecommunication Dept. (DET), Politecnico di Torino, Corso
} Duca degli Abruzzi, 24, Turin 10129, Italy low Earth orbit satellite is the receiving platform. Comparing it with other existing satellite scatterometric, radiometric, and radar applications, GNSS-R remote sensing has several advantages. Firstly, thanks to the global and full-time coverage provided by GNSS satellites, the use of these signals as sources of opportunity allows very dense multi-static radar measurements at L band. Secondly, its passive working principle requires no transmitters except GNSS satellites, thus enabling the system to be light, compact, and cheap. Thirdly, since L band signals are used, the technique works in all-weather conditions and is suitable for altimetric applications (see e.g. $[7,8]$ ) and for sensing nearly all surfaces, such as sea state and wind over sea (see e.g. [9-13]), snow (see e.g. [14-16]), vegetation coverage (see e.g. [17-22]), and soil moisture (see e.g. [23-26]).

A new application based on the possibility of detecting the presence of an object on the terrain or just under it, exploiting the penetration capabilities of electromagnetic energy within the soil, which are inversely proportional to the carrier frequency, is analyzed in this paper. One current application is in the military field, in particular, to detect the presence of improvised explosive devices 
(IEDs) and pressure-activated mines. Mines and IEDs are often hidden on the terrain or inside the vegetation or are buried within the first few centimeters below the surface, since their devastating effects depend of course on their insertion depth.

L band signals (GNSS carrier frequencies are within this band) are not impacted by atmospheric attenuation and normally have a good penetration through vegetation [27]. At $1.5 \mathrm{GHz}$, the penetration depth varies from approximately $10 \mathrm{~cm}$ to $1 \mathrm{~m}$ for soil condition ranging from saturated to dry. In practice, the L band signal can interact with the first $10 \mathrm{~cm}$, depending on the soil moisture level and incidence direction [28,29]. In particular, in the case of almost dry soil, the penetration depth of active systems like GPS or a SAR was found to be around $10 \mathrm{~cm} \mathrm{[30]} \mathrm{or} 7 \mathrm{~cm}$ [31] respectively. Accordingly to [29], for passive $L$ band remote sensing, the penetration depth varies from $10 \mathrm{~cm}$ to $1 \mathrm{~m}$ depending on whether the soil is wet or dry. These values are upper-bound values that can be used when the soil is homogeneous, as in the case of our first experiment (dry or wet sand). With a nonuniform moisture profile, a 'soil moisture sensing depth' definition [29] could be used and its approximation of one tenth of a wavelength in the medium would lead to less than $2 \mathrm{~cm}$ at $1.4 \mathrm{GHz}$. However, the penetration depth is strongly influenced by the soil density, soil moisture, and composition, and many models of soil can be considered and more realistic evaluation performed.

For the detection of mines that are hidden in the superficial layer of the ground (explosive devices are hidden in the first few $\mathrm{cm}$ below the surface in order to make their devastating effects as effective as possible), this penetration capability is enough. Generally, complicated and expensive devices are used to detect explosive objects [32,33]; most of them work very well, but they need the human presence on the field to move the detector.

In this paper, the capability of GNSS-R signals to detect buried metallic objects is investigated through the use of a very simple and low-cost software receiver. This receiver is relatively light and can be mounted on board a remotely controlled unmanned aerial vehicle (UAV), thus avoiding the human presence in the field. The receiver was connected to a left-hand circularly polarized (LH) antenna to collect signals reflected from the ground. Surface roughness was not taken into account and the reflected signal power was estimated considering only coherent power. An open-loop approach was used for deriving signal to noise ratio (SNR) time series related to the reflected GPS signals.

Two prototypes were developed. The first was a software receiver, and the second was a more compact prototype suitable for use on board UAVs based on a
Hackberry board to manage the receiver front end and store the raw data. The post-processing was done using a standard laptop. Several measurement campaigns were carried out with and without a metal object consisting of a metal plate. The first measurement campaign described in this work was performed in static conditions on sandy terrain to check the functionality of the system and the sensitivity of the results to the presence of the metal obstacle. In the second measurement campaign, the antenna moved along a given path, mimicking a flight. The results obtained highlight the possibility of using GNSS-R signals not only to detect buried metallic objects but also to estimate their dimensions.

This paper is organized as follows. In 'Section 2', the microwave properties of soil and the potential of buried object identification are described. In 'Section 3,' the receiver hardware and the signal processing and post-processing are detailed. The various measurement campaigns and results are discussed in 'Section 4,' while in 'Section 5,' our conclusions and future work are highlighted.

\section{Buried object detection capabilities in L band}

The dielectric properties of wet soil have been studied by several authors (e.g. $[27,34])$. These properties depend on water content and soil texture and on the carrier frequency of the signal used for monitoring purposes. The high dielectric constant of water significantly increases both the real and imaginary parts of the soil's dielectric constant as the water volumetric concentration increases. The dependence on soil type (or 'texture') is due to the different percentages of water bound to the surface of the different particles characterizing the soil. Bound water particles exhibit less freely molecular rotation at microwave frequencies and hence are characterized by smaller dielectric effects than the free water in the pore spaces. This is most evident in clay soils, which have greater particle surface areas and affinities for binding water molecules and hence are capable of holding greater percentages of bound water. The dependence of dielectric constant for a sandy soil on the signal carrier frequency is reported in [35]. The real part is almost constant below $5 \mathrm{GHz}$, while the imaginary part is strongly frequency dependent. As reported in [29], this frequency dependence can be taken into account considering the penetration depth which depends on the moisture volumetric concentration and on the wavelength. At the L1 carrier frequency of the GPS signal $(1,575.42 \mathrm{MHz})$, penetration depths decrease from $1 \mathrm{~m}$ to $10 \mathrm{~cm}$, from dry soil to $30 \%$ water concentration. The penetration depth also depends on the elevation angle of the antenna. Since the nadir incidence is the best case, in our experiments, the antenna boresight was aligned very close to the nadir direction (approximately $5^{\circ}$ off the nadir). 


\section{Hardware and signal processing}

The results shown in this paper are based on several measurement campaigns in which an ad hoc receiver based on a software-defined radio solution was used. This system was basically composed of an antenna, a plastic-wood support where the receiving antenna was fixed in order to maintain a specific height from the terrain, a radio frequency front end circuit for the frequency down-conversion of the received signal and for its digital conversion, and a suitable hardware device to store and process the data (see Figure 1).

\subsection{Hardware}

A summary of GPS system characteristics can be found in [36]. Each GPS satellite broadcasts a carrier signal at $1,575.42 \mathrm{MHz}$, referred to as 'L1', modulated by a civilian code (the so-called Coarse Acquisition code). Additionally, another code is broadcasted through a carrier frequency of $1.2276 \mathrm{GHz}$ (L2) for military use, but reception of this signal requires complicated signal processing since it is encrypted. Even if at the time of the experiment few satellites started the transmission of the new civilian L2C signal, all the algorithms were based on the processing of the Coarse Acquisition (C/A) code. Therefore, only the GPS L1 carrier signals were used in our bistatic radar remote sensing experiment. The signals are encoded with timing and navigation information and transmitted with right-

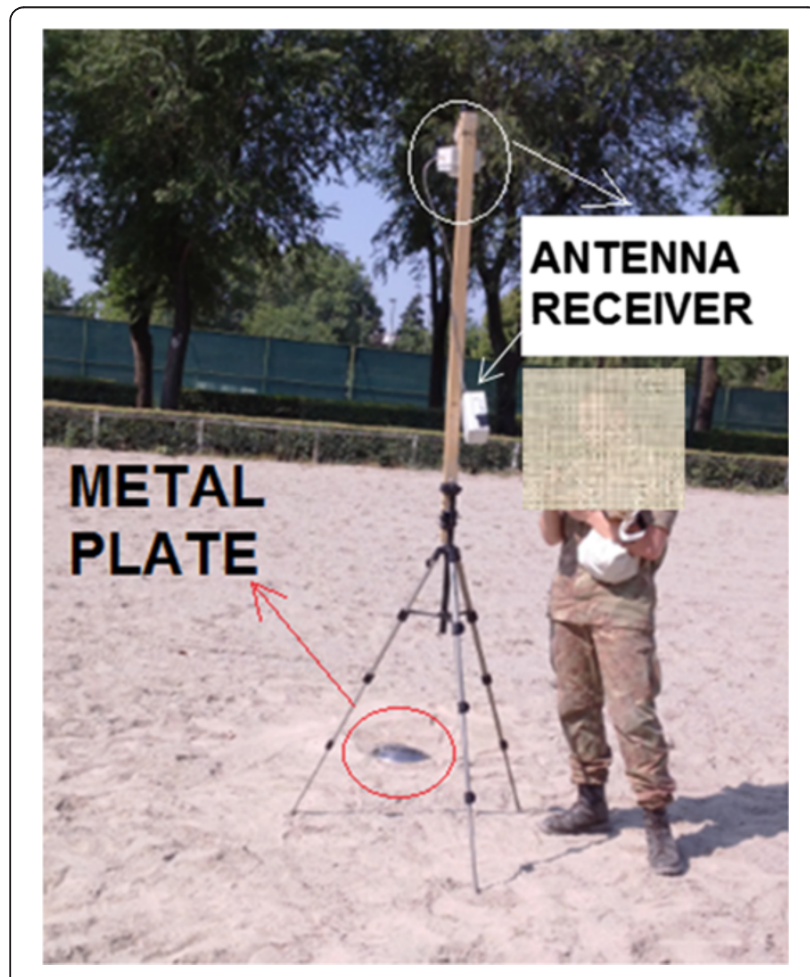

Figure 1 Experiment A: Static measurement setup. Tripod and wood-rod support for the receiver and the antenna. hand circular polarization $(\mathrm{RH})$. The receiver can then calculate the positions of the transmitting satellites and use this information to calculate its own position and GPS time. A low-gain, quasi-hemispherical, zenith patch antenna is normally used to receive the direct signals. The GPS signals are also reflected off by the Earth's surface and can be received by a nadir-viewing antenna at a further delay with respect to the direct signal. After reflection, the scattered signal is predominantly LH for typical incidence far away from the Brewster angle. A low-gain, quasi-hemispherical, LH nadir antenna was used to measure the scattered signal. This antenna was chosen in order to have more flexibility in the measurements of signals characterized by different angles of incidence and because the geometry slowly changes with transmitter and receiver positions. Even if the cross-pol level of our antenna was not very good (approximately $-15 \mathrm{~dB}$ ), the $\mathrm{RH}$ component of the reflected signals (generated by scattering phenomena inside the glistening zone) is expected to be from -10 to $-20 \mathrm{~dB}$ lower than the $\mathrm{LH}$ one. This means that the contribution due to the $\mathrm{RH}$ power available at the output of the LH antenna is a very small (and negligible) fraction of the wanted LH component. Other important figures of merit to be considered for the choice of the antenna are the half-power beamwidth (HPBW; and its projection on the ground, i.e., the antenna footprint) and the entire antenna's radiation pattern. The HPBW should be as wide as possible, in order to be able to contemporaneously acquire as many reflected signals as possible. The signals can then be easily separated on the base of the Pseudo Random Noise (PRN) code modulating the GPS L1 frequency (called C/A code), which uniquely characterizes the transmitted signal.

It has to be noted that only a portion of the footprint will be 'sensitive' to the reflected signal, namely the first Fresnel zone, which is the projection on the ground of the first Fresnel ellipsoid defined considering the geometry and the wavelength [37]. The majority of the reflected power is generated within this area, particularly when the terrain can be considered flat at the used wavelength. If scattering over a rough surface occurs, a wider area (the so-called glistening zone) should be taken into account.

Even though the antenna allows simultaneous reception of both the polarized components of the reflected signal, only the LH one was processed in these experiments. The processing of the $\mathrm{RH}$ component can provide some interesting contribution to minimize surface roughness effects when the goal is to remotely sense some geophysical parameter of the surface. The hypothesis that for moderately rough surface the ratio of two orthogonal polarizations does not depend on the surface roughness was formulated by [38]. Recently, the fact that both reflection coefficients for reflected $\mathrm{LH}$ and $\mathrm{RH}$ are 
sensitive to surface roughness but their ratio is seen to be independent from the roughness was experimentally proved by [23].

In our case, the goal was to detect objects with some metallic part, just under the ground surface. In this situation, the signal received after the scattering from the metallic part is strong enough to be detected even if the object is placed under a very rough surface.

Another important hardware choice concerns the radio frequency front end circuit. The SiGe GN3S Sampler v2, developed from the Colorado Center for Astrodynamics Research, was used [39]. It is composed of two main integrated circuits. The first one is an application-specific integrated circuit (ASIC), which basically amplifies the incoming radio frequency (on the L1 GPS bandwidth), filters it, down-converts it from the GPS carrier frequency to an intermediate frequency of $38.4 \mathrm{MHz}$, and samples it (with a sampling rate $8.1838 \mathrm{MHz}$, which can provide up to eight samples per code chip of the modulating $\mathrm{C} / \mathrm{A}$ code). Two bits for representing both the in-phase and the quadra-phase samples of the signal component are used and are sent to the second circuit, the microcontroller, which transfers in real time the ASIC-generated samples into a USB.

Basically, the system we developed is a softwaredefined radio device. Even if the other steps of signal processing are performed following a pure software approach, a device to store and to post-process all the samples of the raw signal available is necessary. In this case, we developed two different solutions. The first one is based on the use of a laptop PC which is directly connected to the front end through the USB port (see Figure 2, left). This configuration can be easily extended to support two different front ends, one of them connected to an up-looking $\mathrm{RH}$ antenna, in order to allow the collection also of the direct GPS signals for positioning purposes and for georeferencing specular reflection points into the terrain. The direct signal can also be used for calibration purposes. This is an important task to be performed if the goal is to quantitatively extract geophysical parameters from the reflected signal or to have an idea on the Doppler shift characterizing the reflected signal. Also in this case, a software GPS receiver solution can be easily adopted, implementing all the standard closed-loop signal processing steps to the digital samples of the received raw signal (see for example the NGene software receiver, developed by the NAVigation Signal Analysis and Simulation (NAVSAS) group of the Politecnico di Torino, Italy [40]).

A more stand-alone, compact, and trendy solution was based on a system on chip (SoC) device able to store a large amount of raw samples available during a single measurement: the Hackberry A10 Development Board [41], (see details of this board in Figure 3). The entire system was implemented on an electronic board (100 $\mathrm{mm} \times$ $80 \mathrm{~mm})$. Thanks to its lightness, small dimensions, and independency from external power source, it can be easily used as a payload for a small unmanned aerial system (UAS), remotely and/or automatically controlled. Some internal subsystems - including the storage device, the oscillator, the power supply (it can be powered thanks to an external battery which is also able to provide the required current to the antenna's low-noise amplifier), the Ethernet LAN, and the USB management - were customized in order to better suit the performance of the front end. A customized version of the operating system based on Linux Debian to be installed on the Hackberry board processor was also developed. The entire device was able to operate via the $\mathrm{SSH}$ protocol using either the Ethernet LAN port or a wireless connection (an internal Wi-Fi transmitter is available). This is extremely useful in order to access the onboard firmware which contains several user setup parameters.

The board, the front end, and the antenna were finally integrated into a single box, as shown in Figure 2, right. This second solution was adopted from static position only in order to test its effectiveness. After the board is switched on, the operating system boot takes around 80 s. Data can be acquired for a certain user-defined time interval (around $40 \mathrm{~s}$ ) or up to when the onboard
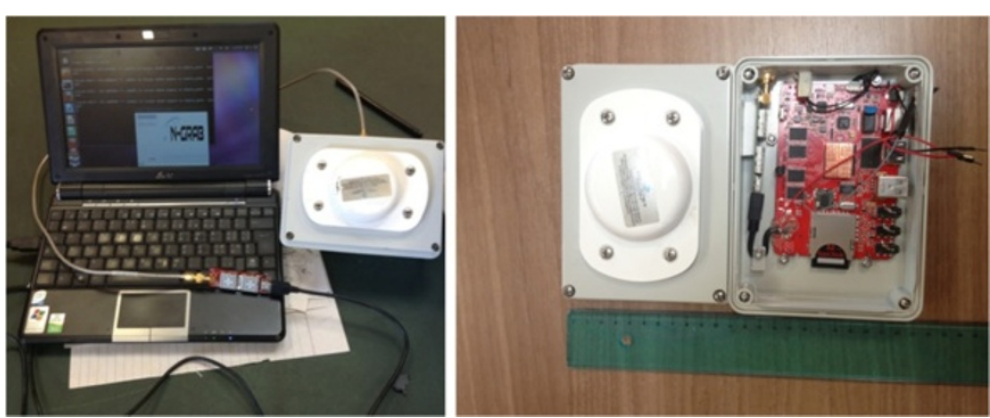

Figure 2 Prototypes of the GNSS-R receivers developed. PC-based solution (left panel). Hackberry board connected to the SiGe radio frequency front end (right panel). 


\begin{tabular}{|c|c|}
\hline System & $\begin{array}{l}\text { 1.2GHz Cortex A8 ARM Core (Running @ 1.0GHz) } \\
\text { - MALI400MP OpenGL ES } 2.0 \text { GPU } \\
\text { DDR3 1333 9-9-9 1GB } \\
\text { 1080p Hardware-accelerated Video playback } \\
\text { 4GB NAND Flash Controller }\end{array}$ \\
\hline yo & $\begin{array}{l}\text { - } 2 \text { SDIO interfaces (SD 3.0, UHI class) are Broken Out } \\
\text { 1 x SD Card Slot } \\
\text { - } 1 \text { x USB 2.0 Downstream Standard Ports via GL850G Host Controller } \\
\text { - } 1 \text { x UART (3.3v TTL 4-pin header) } \\
\text { - } 1 \text { x IR Receiver } \\
\text { - Power Button, Reset Button, Recovery Button }\end{array}$ \\
\hline Digital Audio/Video Output & $\begin{array}{l}\text { - HDMI(1080p) } \\
\text { - HDMIDigital Audio Out }\end{array}$ \\
\hline Analog Audio output & $\begin{array}{l}\text { - } 3.5 \mathrm{~mm} 2 \text { Pole Headphone } \\
\text { - } 3.5 \mathrm{~mm} \text { Mic In }\end{array}$ \\
\hline Analog Video output & $\begin{array}{l}\text { - } 3.5 \mathrm{~mm} \text { Composite } \mathrm{AV} \\
\text { - } 3.5 \mathrm{~mm} \text { Component } \mathrm{Y} / \mathrm{Pb} / \mathrm{Pr}\end{array}$ \\
\hline Power & $\begin{array}{l}\text { - AXP209 PMU } \\
\text { - NEMA 2-pin power adapter included } \\
\text { Input AC100-240V-0.4A } 50 / 60 \mathrm{~Hz} \\
\text { Output DC5v } 1.2 \mathrm{~A} \\
\text { - Battery Functionality is not broken out }\end{array}$ \\
\hline Network Connectivity & $\begin{array}{l}\text { - } 10 / 100 \text { Ethernet (MII compatible) } \\
\text { - RTL8188CUS WiFi b/gn USB Module }\end{array}$ \\
\hline
\end{tabular}

Figure 3 Characteristics of the Hackberry A10 Development Board.

RAM is full. The data acquired are automatically downloaded into an onboard SD card. The performances of the Hackberry A10 Development Board are not adequate for the processing steps explained in 'Section 3.2'; acquired data are currently post-processed by a standard PC.

\subsection{Signal processing and post-processing software}

Data stored on the PC or on the SD card were postprocessed implementing the first signal processing stages for standard open-loop GPS data acquisition using ad hoc MATLAB routines. The open-loop approach was implemented in order to avoid time delays due to the first fix standard procedures and to avoid possible signal loss of lock due to low SNR values [42]. For each available satellite, a software routine dispreads the reflected signal reconstructed from its in-phase and quadra-phase intermediate frequency components. Basically, this standard operation consists in correlating raw data with a replica of the transmitted GPS C/A code locally generated by a software routine, in both delay and Doppler frequency shift domains (see for example [36] for a more detailed discussion of basic GPS signal acquisition and tracking). For each Doppler shift (analyzed with a resolution of $1 \mathrm{kHz}$ or $10 \mathrm{~Hz}$ in a bandwidth of $20 \mathrm{kHz}$ or $200 \mathrm{~Hz}$ around the carrier frequency for the first raw solution or for the more refined one), the correlation was performed in the frequency domain instead of doing it in the more time-consuming time domain. The correlation amplitude is then squared in order to get the correlation power (Figure 4 shows an example). This variable is provided for the entire duration of one GPS C/A code period ( $1 \mathrm{~ms}$ ) with a time resolution provided by the sampling rate of the radio frequency front end (which is $8.1838 \mathrm{MHz}$, i.e., $0.125 \mathrm{C} / \mathrm{A}$ code chip length being the entire code formed by 1,024 chips). This time interval is also known as coherent integration time. In order to improve the SNR, in principle, it is possible to coherently integrate this value considering $20 \mathrm{~ms}$ of data. Longer intervals require knowledge of the navigation data bit hidden in the C/A code sequence. From the delay Doppler map (DDM), the socalled delay waveform (DW; the entire correlation function containing the maximum value evaluated in function of the range) was extracted. Since the reflected signal power is attenuated by the surface scattering process [13], the correlation peak of the reflected signal hardly emerges. Therefore, before extracting DW, a noncoherent integration was performed summing together several consecutive DDMs. The impact of different noncoherent integration time intervals was analyzed in detail, and finally, a 500-ms interval was chosen for processing all the acquired data sets. Further increase of the integration time does not change significantly the final results. Moreover, the integration time cannot be increased too much in applications where the receiver is moving. An example of an integrated DDM is shown in Figure 5, left. There are 8,184 samples in the $x$-axis (one C/A code period) and 20 frequency steps within the range from -10 to $10 \mathrm{kHz}(1-\mathrm{kHz}$ step, $y$-axis). The $z$-axis shows the normalized correlation power. The corresponding DW is shown in Figure 5, right.

A noncoherently integrated peak value is therefore available each $500 \mathrm{~ms}$. Corresponding SNR time series 


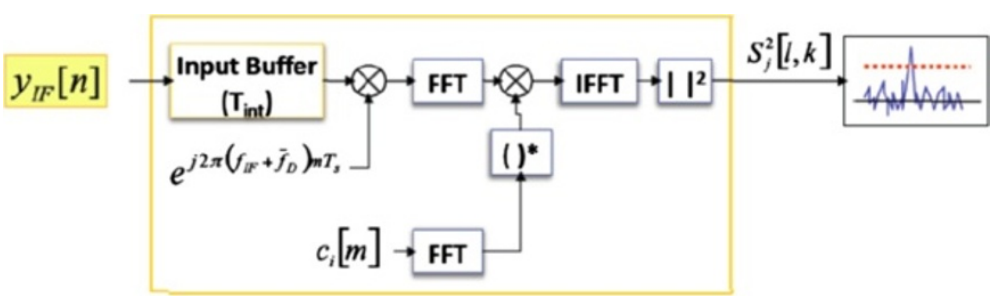

Figure 4 Flow diagram of parallel GPS signal acquisition. $y_{I F}$ is the intermediate frequency input sample sequence, $f_{D}$ is the current Doppler shift, $C_{\mathrm{i}}$ is the locally generated $\mathrm{i}-\mathrm{PRN}$ GPS C/A code, and $S_{\mathrm{j}}$ is the correlation power. $T_{\text {int }}$ defines the coherent integration time $(1 \mathrm{~ms}$, which is the length of one C/A code period).

can therefore be estimated. The SNR of the received signal can be written as

$$
\Phi(\hat{\tau}, \hat{f})=\frac{G_{D} \cdot P_{\mathrm{S}} \cdot \Lambda^{2}(\hat{\tau}-\tau) \cdot S^{2}(\hat{f}-f)}{P_{\mathrm{N}}}
$$

where $P_{\mathrm{S}}$ and $P_{\mathrm{N}}$ are the signal and noise power before despreading, respectively. Ambiguity functions $\Lambda$ and $S$ represent the 'attenuation' due to power correlation misalignments, in delay and frequency, and $G_{D}$ is the socalled processing gain (approximately $30 \mathrm{~dB}$ ) due to the despreading of the GPS C/A code. $P_{N}$ is the input noise power that can be expressed as

$$
P_{\mathrm{N}}=k T_{\mathrm{N}} B_{w}
$$

where $k$ is the Boltzmann constant, $k=1.380 \times 10^{-23} \mathrm{~J} / \mathrm{K}$; $T_{\mathrm{N}}$ is the estimate of the receiver noise equivalent temperature (which can be approximated as $T_{\mathrm{N}}=(N F-1)$ 290), $N F(\mathrm{~dB})$ being the receiver noise figure (it can be estimated in the range of 1.0 to $2.5 \mathrm{~dB}$ ); and $B_{w}=1 / T_{\mathrm{I}}$ is the signal bandwidth determined by the coherent integration time $T_{\mathrm{I}}\left(1 \mathrm{~ms}\right.$ in our case). It results in $P_{\mathrm{N}}=-176.3 \mathrm{~dB} \mathrm{~W}$. The antenna's temperature (TA) was not taken into account in the input noise power evaluation because the measurements were carried out to detect the metallic object and to estimate its dimension by evaluating the relative increase (or decrease) of the SNR, without changing the experimental setup.

The SNR to be estimated is related to the correlation peak available after despreading. Thus, the attenuation factor due to the ambiguity function $\Lambda^{2}(\hat{\tau}-\tau) \cdot S^{2}(\hat{f}-f)$ is close to 1 (the reflected signal is received with a delay $\hat{\tau}$ and Doppler shift $\hat{f}$ ). Finally, the received signal power $P_{\mathrm{S}}$ can be expressed by the following simplified equation derived from Equation 1:

$$
P_{\mathrm{S}}=\frac{\Phi_{\text {peak }} \cdot P_{\mathrm{N}}}{G_{D}}
$$

where $\Phi_{\text {peak }}$ is the absolute signal to noise ratio (ratio between the pure signal and the noise powers) and it can be easily evaluated considering the normalized DW. In particular, the noise floor $\overline{P_{\mathrm{N}}}$ can be estimated as the DW averaged level computed over a region of delays where no signal $\Phi_{\text {peak }}$ is present. Therefore, the SNR obtained by the measurement can be easily derived as follows:

$$
\Phi_{\text {peak }}=\frac{1-\overline{P_{\mathrm{N}}}}{\overline{P_{\mathrm{N}}}}
$$

The estimated total received power $P_{\mathrm{S}}$ (coherent signal power) can be derived from Equation 3. Even if only the

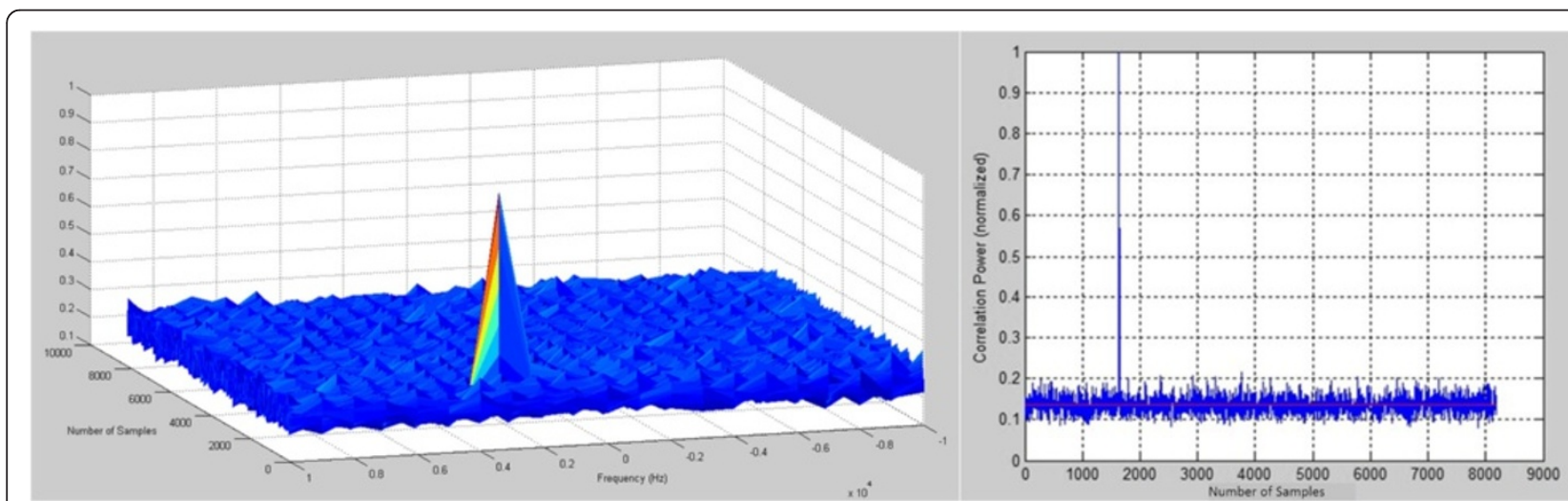

Figure 5 Delay Doppler Map (DDM; left panel) and corresponding delay waveform (DW; right panel). 
value of the correlation peak was used to estimate SNR, this open-loop approach allowed us to develop and implement the software procedure to evaluate the entire autocorrelation function, whose knowledge could be used in the future for other GNSS-R applications, more oriented to the remote sensing of surface parameters. As far as the detection of buried objects is concerned, the estimation of the SNR time series is enough, as it will be discussed in 'Section 5.'

\section{Measurement campaigns and results}

In this section, the results of two experiments performed during the 2013 summer season are discussed:

A. Piazza d'Armi, Turin, Italy, 16 July, 2013, antenna in a static position, compact receiver, sandy terrain

B. Montoro, Avellino, Italy, 22 August, 2013, moving antenna, PC-based receiver, grass terrain

All the experiments were carried out considering as a target a circular metal disk (28-cm diameter) object. The dimensions of this object are comparable to those of an improvised explosive device or a pressure-activable mine.

A MATLAB tool to predict the positions of all the specular reflection points automatically projected on a Google Earth map for any GPS signal available was developed. The specular reflection points can be found on the basis of the receiver position and the predicted GPS satellite orbits (downloaded from CALSKY website www.calsky.com - and based on the predicted IGS orbits). Knowledge of the expected positions of available reflections given by this tool was fundamental for the planning of the measurement campaigns. The antenna used was a commercial device, manufactured by Antcom [43]. It is an active L1/L2 RH/LH antenna (PN 4261215 ), characterized by a HPBW of $140^{\circ}$ (maximum gain $3.5 \mathrm{~dB}$ ). The antenna was fixed on a plastic-wood structure in order to perform the measurements at a constant height $(3 \mathrm{~m})$ from the ground and in far field conditions.

\subsection{Piazza d'Armi experiment (16 July, 2013)}

This experiment was performed in order to evaluate the sensitivity of the system to the presence of a metal object above or just under a dry or a completely wet sandy terrain (the metal plate was buried 5 to $10 \mathrm{~cm}$ under the surface). The compact receiving solution was used. The antenna was mounted at $3 \mathrm{~m}$ height from the ground on top of a wooden rod fixed to a static tripod. The antenna beam axis was moved $5^{\circ}$ away from the nadir position in order to avoid interference with the tripod structure and with the receiver itself, which was fixed to the same wooden rod (see Figure 1). The experiment geometry was carefully designed considering the prediction of the specular reflection point positions (see Figure 6). The positions of each specular reflection point, estimated considering GPS orbits predicted in advance (taken by the International GNSS Service website) with a sample time of $5 \mathrm{~min}$ for an overall experiment length of $50 \mathrm{~min}$, are shown for each reflected signal (coming from different GPS satellites). Blue ellipses depict the first Fresnel zone boundaries evaluated from geometry for the first specular point of each series (identified by the bold colored specular reflection point). The antenna footprint is defined by the black line. The plot shows distances in meters, considering the receiving antenna (pink dot) in the origin of the reference system.

The Google Maps is shown in Figure 7, while the estimated positions of the specular points are shown in Figure 6. The sample time of each point in Figure 6 was $5 \mathrm{~min}$, and 10 positions for each specular reflection point were plotted, based on predicted orbits. We started the simulation at 2:40 p.m., and we ended the prediction after 50 min (10 different specular reflection points are therefore shown). The experiment started at 2:55 p.m. and lasted $20 \mathrm{~min}$. This means that the predicted reflection point positions during the experiment are from the third to the eighth point (the Fresnel zones should be shifted). Five continuous 40-s raw data time series were taken, and the configuration of the target and of the surrounding terrain was changed (leaving the metal plate always in the same position with respect to the antenna's boresight). In particular, the following five time slots were considered:

A1 - from 2:55 to 2:56 p.m. (local time), the metallic plate was placed on dry soil far away from the expected first Fresnel zone.

A2 - from 3:00 to 3:01 p.m., the metallic plate was removed from the antenna footprint.

A3 - from 3:09 to 3:10 p.m., the metallic plate was buried under the dry soil.

A4 - from 3:12 to 3:13 p.m., the metallic plate was placed on dry soil.

A5 - from 3:14 to 3:15 p.m., the metallic plate was buried under completely wet soil.

The five SNR time series coming from GPS Space Vehicle PRN 25 (the satellite interacting with the metal plate, as shown in Figure 8) were connected, and the overall trace is shown in Figure 8. For each time series, some statistical indicators are summarized in Table 1.

The first two measurements (A1 and A2) were performed to verify the correct operation of the software receiver in terms of data acquisition. In the first one (A1), the metal plate was not inside the first Fresnel zone, but it was in the antenna footprint. Therefore, the SNR 


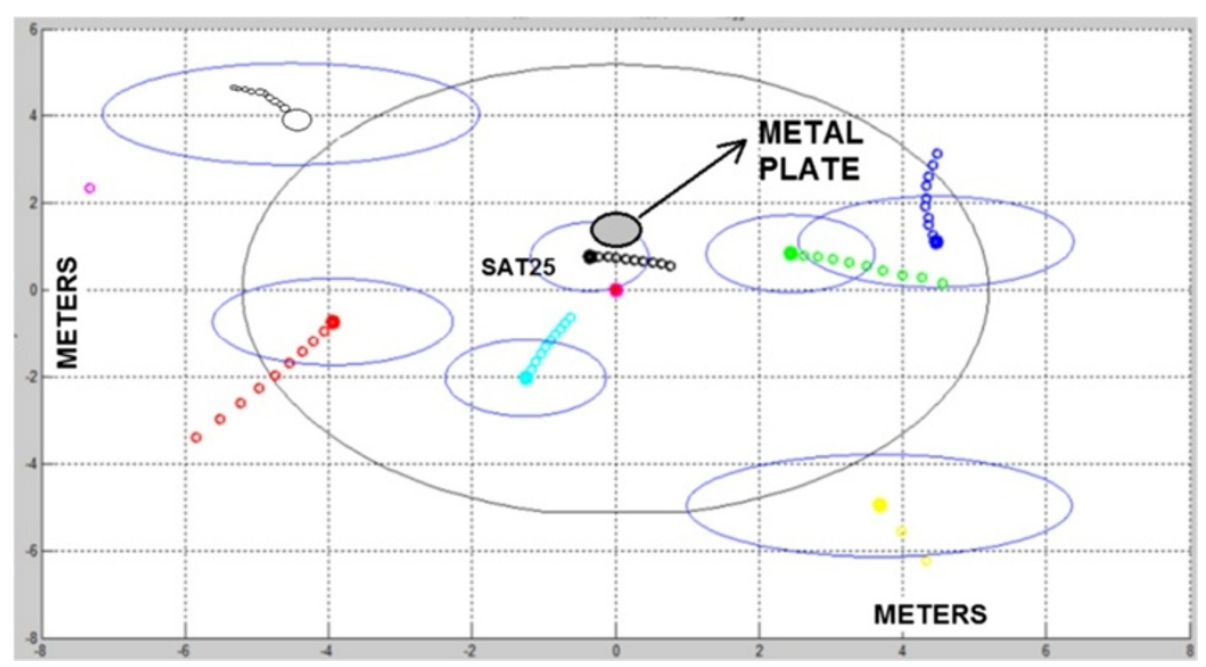

Figure 6 Experiment A: Prediction of reflection points on a $(x, y)$ plane. Different colors depict signals coming from different GPS satellites. The black ellipse is the antenna's footprint. Blue ellipses depict the first Fresnel zone boundaries evaluated from geometry for the first specular point of each series (identified by the bold colored specular reflection point). Each specular point position has been evaluated considering predicted GPS orbits. The plot shows distances in meters, considering the receiving antenna (pink dot) in the origin of the reference system.

estimate of $+2 \mathrm{~dB}$ (mean value) also takes into account some of the power scattered out from the specular direction by the metal plate. During the second time slot (A2), the object was removed, but an unexpected event occurred in the receiver hardware around the 200th sample. In this case, a more realistic statistical figure for the estimated SNR would be around $-1 \mathrm{~dB}$ (also the std figure shown in Table 1 is not representative). The presence of the metallic plate over dry soil (A4) or just buried under it (A5) produces a significant increase in the received power (from around $-1 \mathrm{~dB}$ without any object to 5 or $7 \mathrm{~dB}$ ). This increase in the SNR should be produced by the metallic object only, since the ground in the (coherent) Fresnel zone (and in the noncoherent glistening - zone) did not change. In conclusion, in the case of dry terrain, where the penetration depth allows more electromagnetic energy to reach the metal plate and to be reflected back towards the receiver, a good sensitivity of the receiver was observed. In fact, a level of $5.1 \pm 1 \mathrm{~dB}$ was measured when the metal plate was buried under the sand, while there was a stronger $6.9 \pm$ $1.3 \mathrm{~dB}$ when it was simply placed above the sand.

A noticeable increase of a further $5 \mathrm{~dB}$ was observed in the case of completely wet sand (A5). This higher contribution to the received power is probably due to the increase of the dielectric constant of the terrain due to the presence of water. Several experiments were done before (but not reported here), but in all of them, the increase of the real part of the dielectric constant due to the water content strongly impacts the detection capability of the receiver.

\subsection{Montoro experiment (22 August, 2013)}

Since the received signal was proved to be sensitive for buried object detection, in this second experiment, the

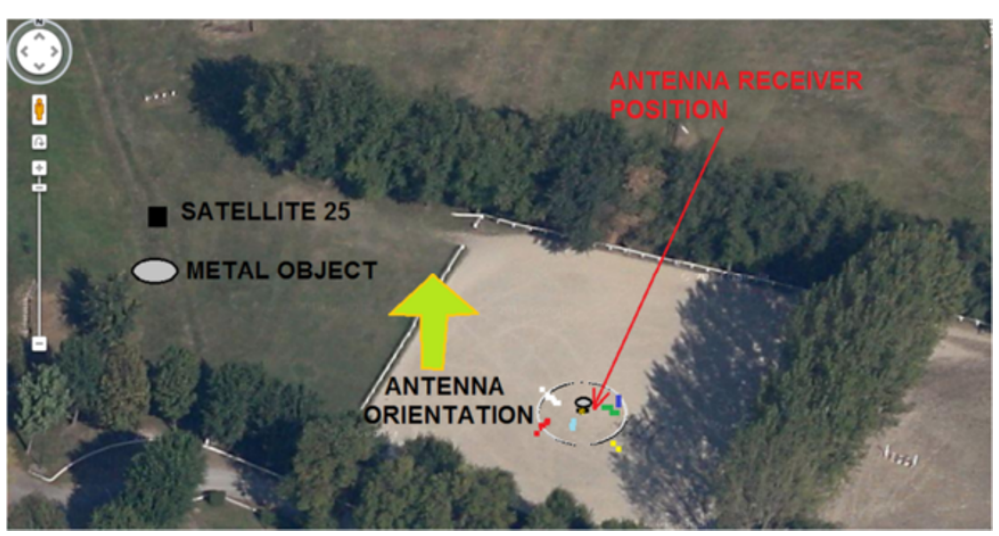

Figure 7 Experiment A: Prediction of reflection points on Google Maps. 


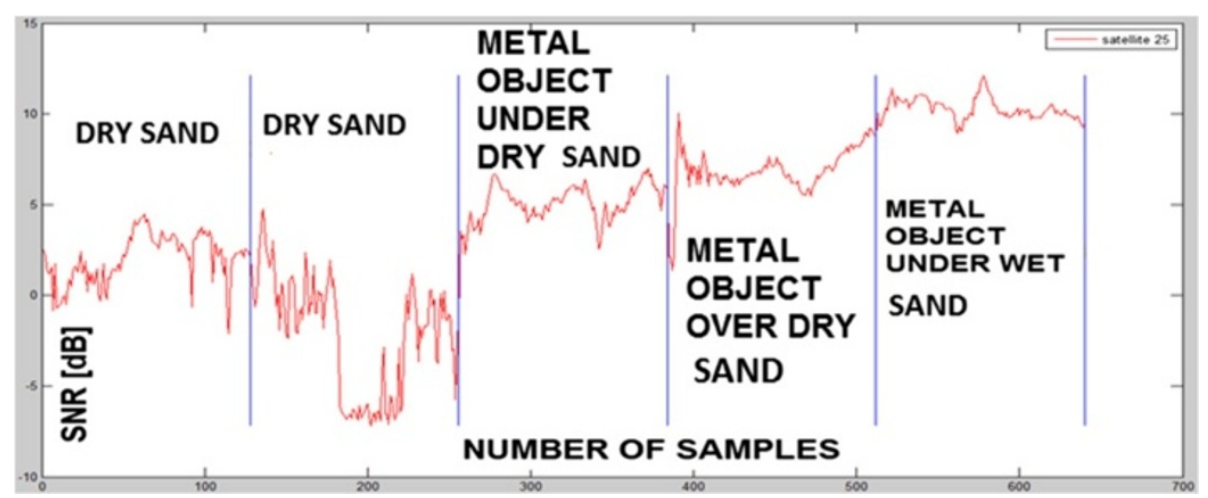

Figure 8 Experiment A: SNR time series for PRN 25.

evaluation of the dimension of the metal plate was investigated by setting up a moving experiment. A kind of unmanned aircraft System (UAS), flying $2.5 \mathrm{~m}$ over a 6m strip of terrain, was simulated as shown in Figure 9. Two plastic boxes forming the receiver support were joined together and fixed to two pulleys between two trees on the terrain. A cable allowed the receiver support to be moved along a rectilinear path. In this second experiment, the PC-based configuration was used in order to continuously store the 40 -s data dumps, avoiding the dead time intervals necessary for the board bootstrap. The prediction of the reflection points for this experiment is shown in Figure 10.

The metal plate was positioned $5 \mathrm{~m}$ away from the starting point ( $1 \mathrm{~m}$ away from the ending point) on a portion of ground on which a contribution to the reflection of the signal coming from PRN 24 was expected.

In this case, the effects due to vegetation canopy and grass coverage should be taken into account. The estimation of the quantitative impact is very difficult, being a combination of incidence angle, wavelength, biomass volume, height, and loss component induced by the dielectric constant of water-containing stalks and leaves. In addition to the theoretical approach described by Ulaby et al. (see $[44,45])$, a detailed analysis is presented in [22,23]. As a first approximation, an average reduction of the SNR of $2 \mathrm{~dB}$ due to the effect of vegetation will be taken into account.

Three 'flights' were performed:

B1 - from 8:50 to 8:51 a.m. without the 28-cm-diameter metal plate
B2 - from 8:52 to 8:53 a.m., with the metal plate placed on the soil

B3 - from 8:54 to 8:55 a.m., with the metal plate buried approximately $4 \mathrm{~cm}$ under the soil

As expected, a strong 'dynamic' on the SNR time series (approximately $8 \mathrm{~dB}$ in this case, from $-6 \mathrm{~dB}$ to around $2 \mathrm{~dB}$ ) was estimated when reflections occurred with or without the metallic plate, for the signal corresponding to PRN 24. In Figure 11, the red plot is the time series evaluated without any object (during the first flight), while the green and the blue lines represent the results obtained when the metal plate was placed above and under the terrain (second and third flight). A difference of approximately $2 \mathrm{~dB}$ between these two cases was estimated. This result is coherent with the SNR difference experienced in the first experiment when the metal plate was moved from the ground below it. The approximately 5-dB increase observed around the 20th sample in the SNR, estimated when the object was placed on the soil (green line), was unexpected. Since the receiver was manually moved, this signal could be related to some strong oscillation caused by the payload 'pulling.' Another important aspect to be taken into account is that the signal due to the presence of the metallic plate was expected to rise between 30 and $40 \mathrm{~s}$. In fact, a mean velocity of $10 \mathrm{~cm} / \mathrm{scan}$ was estimated (the entire 6-m track was completed in approximately $57 \mathrm{~s}$ for all the experiments), and the object placed at approximately $5 \mathrm{~m}$ from the starting point and the first Fresnel zone dimension was approximately equal to $1.5 \mathrm{~m}$, as shown in

Table 1 Statistical characterization of the SNR estimates

\begin{tabular}{ccccccc}
\hline & $\begin{array}{c}\text { A1 - metal object on dry } \\
\text { sand not in the Fresnel zone }\end{array}$ & A2 - dry sand & $\begin{array}{c}\text { A3 - metal object } \\
\text { under dry sand }\end{array}$ & $\begin{array}{c}\text { A4 - metal object } \\
\text { over dry sand }\end{array}$ & $\begin{array}{c}\text { A5 - metal object } \\
\text { under wet sand }\end{array}$ \\
\hline PRN 25 & Median & 2 & -1 & 5 & 6 & 10 \\
& Mean & 2 & -2 & 5 & 6 & 10 \\
\hline
\end{tabular}




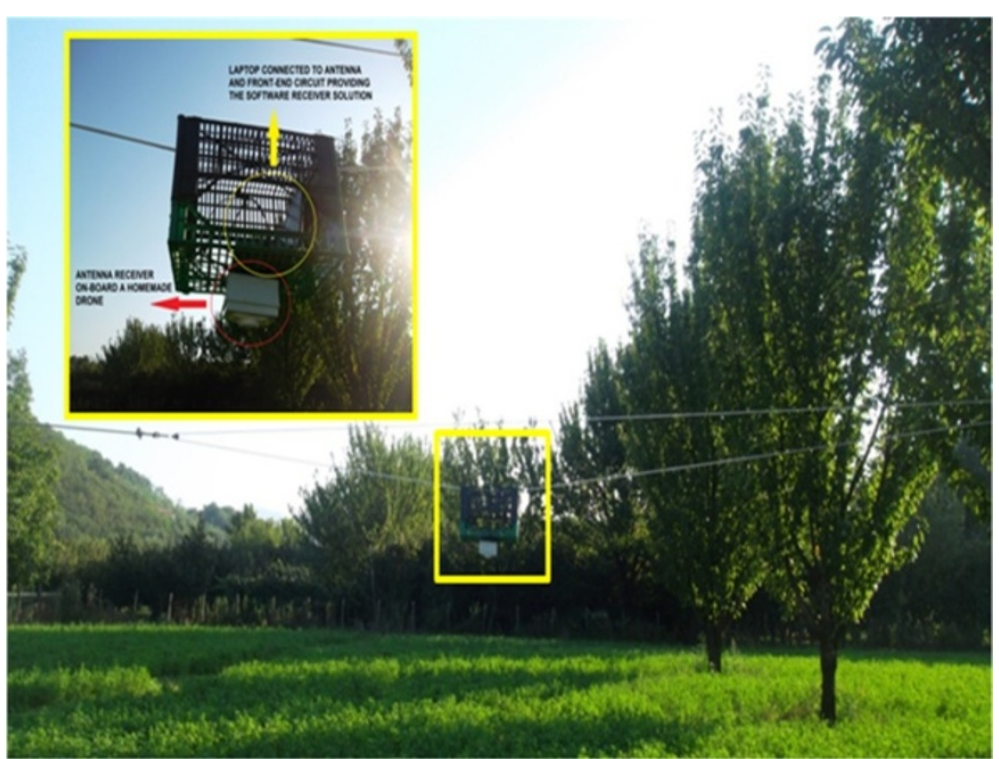

Figure 9 Moving measurement setup for experiment B.

Figure 10. The times when the signal started to rise are identified with the green and blue points in the time series shown in Figure 11. They were computed considering the time when the signal increased by $3 \mathrm{~dB}$ from the background value. The rising time of $35 \mathrm{~s}$ is quite correct for the experiment performed with the metal plate over the soil (green line). For the other case (blue line), the payload velocity during the first half of the experiment was probably greater than that during the second part. Also in this second example, an approximately 2 - $\mathrm{dB}$ difference in the maximum signal available after reflection from the metal plate placed over (green line) or buried in (blue line) the soil was detected.
Thanks to this experiment, an estimate of the dimension of the metal plate was also possible. Since the SNR is related to the energy coherently reflected by the presence of a metal object inside a moving first Fresnel zone, the corresponding time series must be related to the spatial convolution between the Fresnel Zone and the area of the target. In fact, as expected, a trapezoid-shaped SNR time series was observed. As the metal plate was smaller than the dimension of the first Fresnel zone, it is clear that the overall rising time is related to the object dimension (the diameter of the metal plate). This rising time can be empirically evaluated considering the time the SNR needs to increase from a minimum of $+3 \mathrm{~dB}$ to a maximum of $-3 \mathrm{~dB}$.

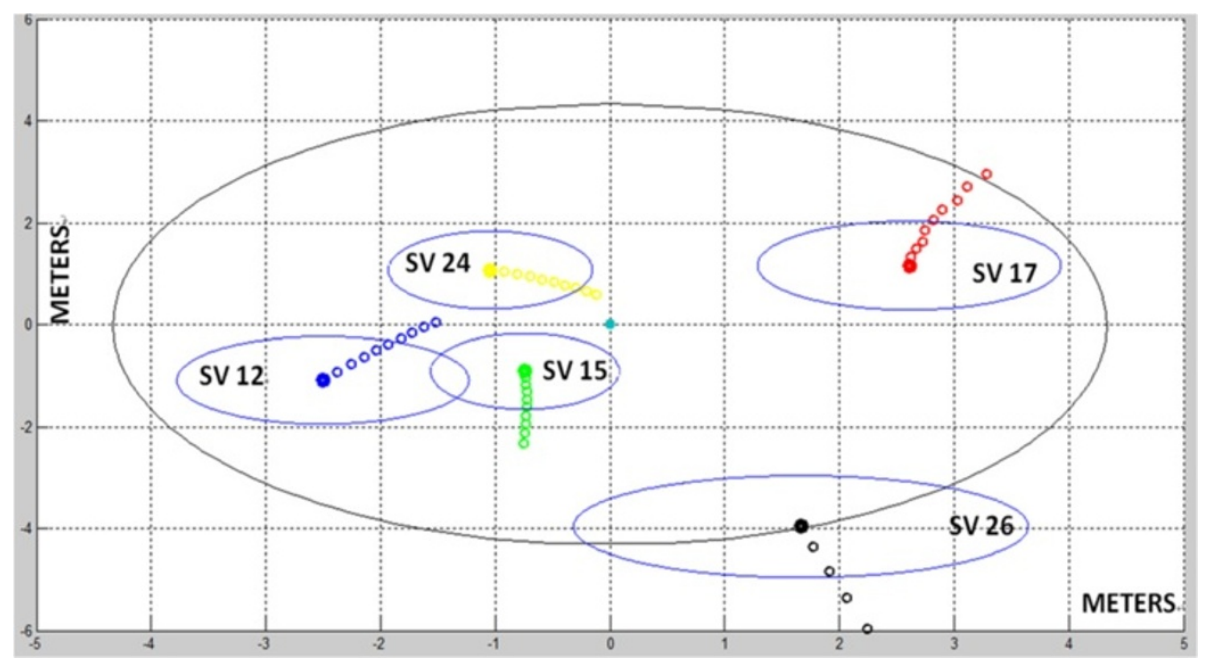

Figure 10 Experiment B: Prediction of reflection points on $(x, y)$ plane. 


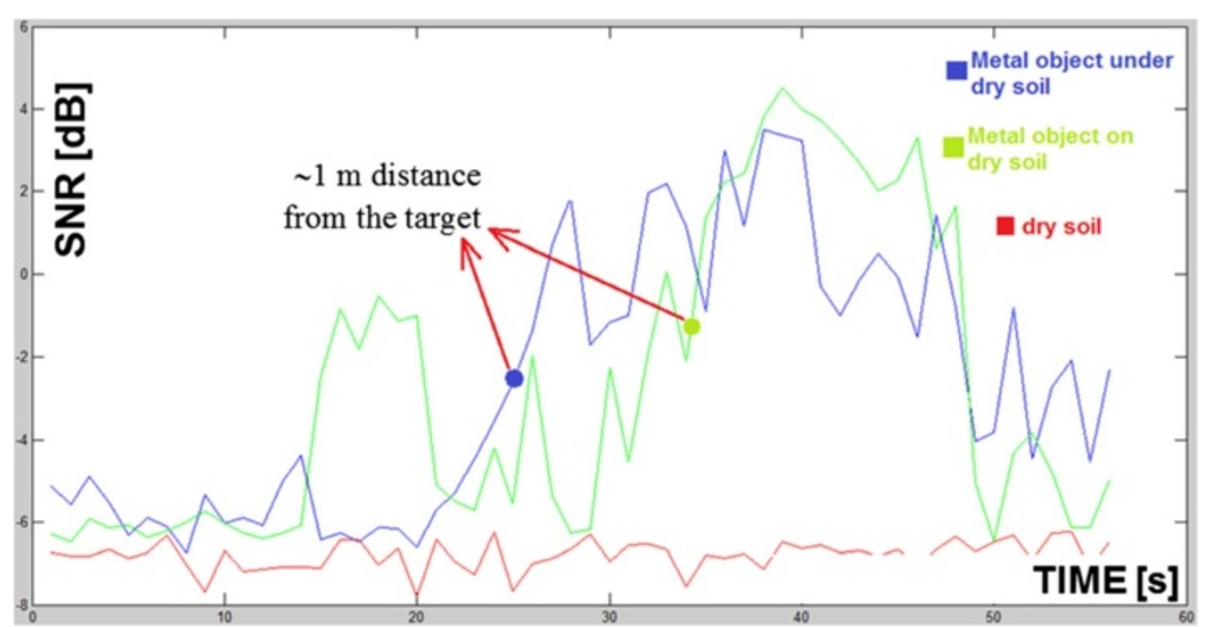

Figure 11 Experiment B: Time series of SNR. Red line is the time series evaluated without any object (first flight), green line the metal plate on the terrain (second flight), and blue lines the metal plate under the terrain (third flight).

In Figure 12, this concept is highlighted on the time series evaluated for the object over the soil (green line in Figure 11), for which a rising time of approximately $3 \mathrm{~s}$ can be identified. Considering an average payload speed of $0.1 \mathrm{~m} / \mathrm{s}$, the dimension of the target can be estimated to be approximately $30 \mathrm{~cm}$ which is close to the diameter of the metal plate.

\section{Conclusions}

In this paper, a new application of GNSS-R technique for the detection of buried objects was investigated. A LH antenna was used to collect reflected GPS signals by a software-defined radio GPS receiver. The effects of surface roughness and vegetation canopy were neglected and the reflected signal power estimated considering only coherent power. An open-loop approach was used for deriving the SNR time series related to the reflected GPS signals.
Two prototypes were developed. The first was a software receiver, and the second a more compact prototype suited for onboard UAV applications. A Hackberry board was used to manage the receiver front end and to store the raw data. The post-processing was done using a standard laptop. Two measurement campaigns were carried, out and the variation of the SNR level due to the presence of a metallic object was investigated. The first measurement campaign was performed in a static condition on a sandy terrain to check the functionality of the system. Note that the presence of the metallic object was detected also in the case of wet terrain. In this case, the effect due to the increase of the dielectric constant characterizing the ground may hide the effect derived from the metallic object. In the second measurement campaign, the antenna was moving along a given path and the possibility of detecting the object dimensions

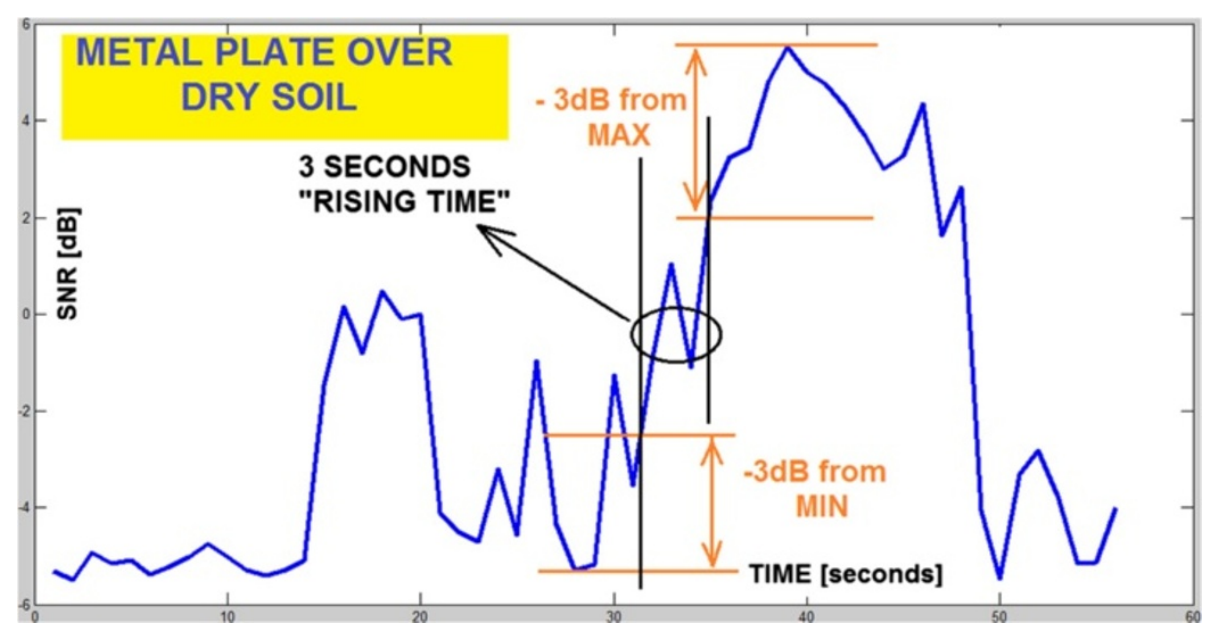

Figure 12 Experiment B: SNR time series evaluated for the object on the soil (green line in Figure 11). 
was highlighted. The results show the possibility of adopting this technique on board an UAV, remotely controlled. In this case, the flying direction could be modified in order to better understand the position and shape of the object. Some other measurement campaigns are foreseen during the forthcoming seasons. In order to remove the strong assumption of a smooth terrain, a new prototype capable of collecting reflected signals on both the polarizations ( $\mathrm{LH}$ and $\mathrm{RH}$ ) is going to be developed and a deeper analysis on the effect of vegetation canopy and its water content, terrain texture, and composition will be addressed in a future work.

\section{Competing interests}

The authors declare that they have no competing interests.

\section{Authors' contributions}

RN conceived the study and participated in its realization and coordination. SDM carried out the measurement campaign and data processing. MC participated in the analysis of the measured data. YP participated in the first measurement campaign. PS participated in the first measurement campaign, the analysis of measured data, and in the realization and coordination of this study. All authors read and approved the final manuscript.

\section{Received: 20 December 2013 Accepted: 14 August 2014} Published: 27 August 2014

\section{References}

1. R Notarpietro, M Cucca, S Bonafoni, GNSS Signals, A Powerful Source for Atmosphere and Earth's Surface Monitoring, in Remote Sensing of Planet Earth (InTech, Yann Chemin, Croatia, 2012), pp. 171-200. ISBN ISBN 9789533079196

2. YA Liou, AG Pavelyev, SS Matyugov, IO Yakovlev, J Wickert, Radio Occultation Method for Remote Sensing of the Atmosphere and lonosphere (InTech, Vukovar, Croatia, 2010), p. 176

3. SG Jin, A Komjathy, GNSS reflectometry and remote sensing: new objectives and results. Adv. Space Res. 46(2), 111-117 (2010). doi:10.1016/j. asr.2010.01.014

4. SG Jin, E Cardellach, F Xie, GNSS Remote Sensing: Theory, Methods and Applications (Springer, 2014)

5. M Martin-Neira, A passive reflectometry and interferometry system (PARIS): application to ocean altimetry. ESA J. 17, 331-355 (1993)

6. JL Garrison, SJ Katzberg, Ml Hill, Effect of sea roughness on bistatically scattered range coded signals from the global positioning system. Geophys. Res. Lett. 25, 2257-2260 (1998)

7. G Ruffini, F Soulat, M Caparrini, O Germain, M Martin-Neira, The Eddy Experiment: accurate GNSS-R ocean altimetry from low altitude aircraft. Geophys. Res. Lett. 31, L12306 (2004)

8. A Rius, E Cardellach, M Martín-Neira, Altimetric analysis of the sea-surface GPS reflected signals. IEEE Trans Geoscience and Remote Sensing. 48, 2119-2127 (2010)

9. JL Garrison, SJ Katzberg, The application of reflected GPS signals to ocean remote sensing. Remote Sens. Environ. 73, 175-187 (2000)

10. ST Gleason, S Hodgart, S Yiping, C Gommenginger, S Mackin, M Adjrad, M Unwin, Detection and processing of bistatically reflected GPS signals from low earth orbit for the purpose of ocean remote sensing. IEEE Trans Geoscience and Remote Sensing. 43, 1229-1241 (2005)

11. N Valencia, A Camps, N Rodriguez-Alvarez, H Park, I Ramos-Perez, Using GNSS-R imaging of the ocean surface for oil slick detection. IEEE J Selected Topics in Appl Earth Observ and Remote Sensing. 6, 217-223 (2013)

12. JL Garrison, A Komjathy, VU Zavorotny, SJ Katzberg, Wind speed measurement using forward scattered GPS signals. IEEE Trans Geoscience and Remote Sensing. 40, 50-65 (2002)

13. ST Gleason, Remote sensing of ocean, ice and land surfaces using bistatically scattered GNSS signals from low earth orbit. PhD thesis (University of Surrey, Surrey, UK, 2006)

14. N Rodriguez-Alvarez, A Aguasca, E Valencia, X Bosch-Lluis, A Camps, I Ramos-Perez, H Park, M Vall-llossera, Snow thickness monitoring using GNSS measurements. IEEE Trans Geoscience and Remote Sensing Letters. 9, 1109-1113 (2012)

15. N Najibi, SG Jin, Physical reflectivity and polarization characteristics for snow and ice-covered surfaces interacting with GPS signals. Remote Sens. 5(8), 4006-4030 (2013). doi:10.3390/rs5084006

16. MD Jacobson, Estimating snow water equivalent for a slightly tilted snow-covered prairie grass field by GPS interferometric reflectometry. EURASIP J Adv Signal Process. 3, 31-41 (2012)

17. EE Small, KM Larson, JJ Braun, Sensing vegetation growth with reflected GPS signals. Geophys. Res. Lett. 37, L12401 (2010)

18. XR Wu, SG Jin, GNSS-Reflectometry: Forest canopies polarization scattering properties and modeling. Adv. Space Res. (2014). 10.1016/j.asr.2014.02.007

19. N Rodriguez-Alvarez, X Bosch Lluis, A Camps, A Aguasca, M Vall llossera, E Valencia, I Ramos Perez, H Park, Review of crop growth and soil moisture monitoring from a ground based instrument implementing the interference pattern GNSS-R technique. Radio Sci. 46, 03 (2011). doi:10.1029/2011RS004680

20. P Liang, LE Pierce, M Moghaddam, Radiative transfer model for microwave bistatic scattering from forest canopies. IEEE Trans Geoscience and Remote Sensing. 43, 2470-2483 (2005). No. 11

21. P Ferrazzoli, $L$ Guerriero, R Rahmoune, Recent Advances in Theoretical Studies of L-Band, Active and Passive Remote Sensing of Forests, in IEEE International Geoscience and Remote Sensing Symposium (IGARSS) (IEEE, Honolulu, Hi, 2010), pp. 3244-3246. doi:10.1109/IGARSS.2010.5651559

22. P Ferrazzoli, L Guerriero, N Pierdicca, R Rahmoune, Forest biomass monitoring with GNSS-R: theoretical simulations. Advances in Space Research. (2010). 10.1016/j.asr.2010.04.025

23. A Egido, M Caparrini, G Ruffini, S Paloscia, E Santi, L Guerriero, N Pierdicca, N Floury, Global navigation satellite systems reflectometry as a remote sensing tool for agriculture. Remote Sensing. 4, 2356-2372 (2012)

24. N Rodriguez-Alvarez, A Camps, M Vall-llossera, X Bosch-Lluis, A Monerris, I Ramos-Perez, E Valencia, JF Marchan-Hernandez, J Martinez-Fernandez, G Baroncini-Turricchia, C Perez-Gutierrez, N Sanchez, Land geophysical parameters retrieval using the interference pattern GNSS-R technique. IEEE Trans Geoscience and Remote Sensing. 49, 71-84 (2011)

25. S Katzberg, O Torres, M Grant, D Masters, Utilizing calibrated GPS reflected signals to estimate soil reflectivity and dielectric constant: results from SMEX02. Remote Sens. Environ. 100, 17-28 (2005)

26. D Masters, P Axelrad, S Katzberg, Initial results of land-reflected GPS bistatic radar measurements in SMEX02. Remote Sens. Environ. 92, 507-520 (2004)

27. J Wang, T Schmugge, An empirical model for the complex dielectric permittivity of soils as a function of water content. IEEE Trans Geoscience and Remote Sensing. 18, 288-295 (1980)

28. E Njoku, P O'Neill, Multifrequency radiometer measurements of soil moisture. IEEE Trans Geoscience and Remote Sensing. 20, 468-475 (1982)

29. EG Njoku, D Entekhabi, Passive microwave remote sensing of soil moisture. J Hydrol. 184, 101-129 (1996)

30. KM Larson, JJ Braun, EE Small, VU Zavorotny, ED Gutmann, AL Bilich, GPS multipath and its relation to near-surface soil moisture content. IEEE J Selected Topics in Applied Earth Observations and Remote Sensing. 3, 91-99 (2010)

31. M Nolan, DR Fatland, Penetration depth as a DInSAR observable and proxy for soil moisture. IEEE Trans Geoscience and Remote Sensing. 41, 532-537 (2003)

32. S Perrin, E Duflos, P Vanheeghe, A Bibaut, Multisensor fusion in the frame of evidence theory for landmines detection. IEEE Trans System, Man and Cybernetics-Part C Appl Rev. 34(4), 485-498 (2004)

33. F Frezza, P Martinelli, L Pajewski, G Schettini, Short-pulse electromagnetic scattering by buried perfectly conducting cylinders. IEEE Geosci. Remote Sens. Lett. 4(4), 611-615 (2007)

34. MC Dobson, FT Ulaby, Microwave dielectric behavior of wet soil - part II: dielectric mixing models. IEEE Trans Geoscience and Remote Sensing. GE-23(1), 35-46 (1985)

35. EG Njoku, JA Kong, Theory for passive microwave remote sensing of near-surface soil moisture. J. Geophys. Res. 82, 3108-3118 (1977)

36. P Misra, P Enge, Global Positioning System: Signals, Measurements and Performance (MA: Ganga-Jamuna Press, Lincoln, 2001)

37. in Propagation of Short Radio Waves, ed. by DE Kerr (Peter Peregrinus Ltd, London, 1987)

38. VU Zavorotny, A Voronovich, Scattering of GPS signals from the ocean with wind remote sensing application. IEEE Trans Geoscience and Remote Sensing. 38, 951-964 (2000). no. 2 
39. GNSS @ Colorado Center for Astrodynamics Research, 2011. http://ccar. colorado.edu/gnss/, Accessed 23 Apr 2011

40. M Fantino, A Molino, P Mulassano, M Nicola, M Pini, N Gene, A GPS and Galileo fully software receiver, in Proceedings of SDR-Italy '09 Workshop From Software Defined Radio to Cognitive Networks (Pisa, Italy, 2009), pp. 2-3

41. Developer Shop by Miniand, Hackberry A10 Dev Board, 2012. https://www. miniand.com/products/Hackberry\%20A10\%20Developer\%20Board, Accessed 20 Sep 2012

42. Y Pei, R Notarpietro, P Savi, M Cucca, F Dovis, A fully software GNSS-R receiver for soil monitoring. Int J Remote Sensing. 35(6), 2378-2391 (2014)

43. Antcom Corperation, 2008. www.antcom.com. Accessed 12 Feb 2013

44. FT Ulaby, RK Moore, TJ Fung, Microwave remote sensing: active and passive From Theory to Application, Vol. II, III (Artech House, Dedham, MA, 1986)

45. FT Ulaby, EA Wilson, Microwave attenuation properties of vegetation canopies. IEEE Trans Geoscience And Remote Sensing. GE-23, 5 (1985)

doi:10.1186/1687-6180-2014-132

Cite this article as: Notarpietro et al:: Detection of buried objects using reflected GNSS signals. EURASIP Journal on Advances in Signal Processing 2014 2014:132

\section{Submit your manuscript to a SpringerOpen ${ }^{\circ}$} journal and benefit from:

- Convenient online submission

- Rigorous peer review

- Immediate publication on acceptance

- Open access: articles freely available online

- High visibility within the field

- Retaining the copyright to your article 